\title{
An Incremental Method for Registering Electroanatomic Mapping Data to Surface Mesh Models of the Left Atrium
}

\author{
Aditya B. Koolwal ${ }^{1}$, Federico Barbagli ${ }^{2,3}$, Christopher R. Carlson ${ }^{3}$, \\ and David H. Liang ${ }^{4}$ \\ ${ }^{1}$ Department of Mechanical Engineering, Stanford University, USA \\ 2 Department of Computer Science, Stanford University, USA \\ ${ }^{3}$ Hansen Medical, Inc., Mountain View, CA, USA \\ ${ }^{4}$ Division of Cardiovascular Medicine, Stanford University, USA
}

\begin{abstract}
We present a method for registering position and orientation data collected from an electroanatomic mapping system (EMS) to a surface mesh based on segmented Computed Tomography (CT) or Magnetic Resonance (MR) images of the left atrium. Our algorithm is based on the Unscented Particle Filter (UPF) for stochastic state estimation. Using an intracardiac echo (ICE) ultrasound catheter with mounted mapping sensor, we acquire ultrasound images of the atrium from multiple configurations and iteratively determine the catheter's pose with respect to anatomy. After considering less than a minute's worth of ICE data, the algorithm converges to an accurate pose estimate which, in turn, yields the registration parameters transforming EMS coordinates to mesh coordinates. The iterative framework of the UPF allows us to be robust to unmodeled EMS noise and drift, problems which complicate traditional registration methods assuming regularity in image data structure.
\end{abstract}

\section{Introduction}

Minimally Invasive Surgery (MIS) techniques have revolutionized cardiovascular therapeutics, enabling physicians to operate inside the anatomy without having to perform open heart surgery. Unfortunately MIS procedures can be very timeconsuming, in part due to difficulties with visualizing the anatomy.

Traditional imaging modalities for guiding cardiac procedures - namely $\mathrm{x}$ ray fluoroscopy and ultrasound - do not adequately capture the anatomy's 3D structure at a high-resolution. A number of augmented, sensor-based solutions have been proposed for guiding catheter ablation treatment of atrial fibrillation \begin{tabular}{lllll}
\hline 1 & 2 & 3 & 4
\end{tabular} . The most widely adopted method employs EMS to record the 3D position and orientation of the ablation catheter's tip as it moves inside the atria. A $3 \mathrm{D}$ model of the atria is generated by plotting the convex hull of all recorded tip position, and physicians use this atrial "point cloud" as an anatomic reference for guidance. Alternatively, the point cloud can be registered to a CT/MR-based surface mesh model, and future recorded tip positions can be subsequently displayed inside this model [34. The latter method has the advantage of highlighting the anatomy's 3D structure in detail.

D. Metaxas et al. (Eds.): MICCAI 2008, Part II, LNCS 5242, pp. 847 854, 2008.

(C) Springer-Verlag Berlin Heidelberg 2008 
A major problem with EMS is that patient motion can move the reference electrode, shifting the coordinates of all recorded points. Should this occur, the process of building up the atrial point cloud must be repeated from scratch. This can be frustrating as data collection often takes up to an hour, and is complex enough to warrant constant supervision. Recent studies have shown the potential of using ICE ultrasound in combination with EMS to speed up the process of acquiring data for $\mathrm{CT} / \mathrm{MR}$ registration [124]. The issue of how to deal with reference electrode movement remains unresolved, although reacquiring data is reported to be substantially less time consuming using this approach 4 .

We propose an incremental approach to the problem of registering EMS data to CT/MR-based surface mesh models. We use ICE ultrasound to speed up the data collection process, and continuously update our registration estimate as new ultrasound images are considered. Each acquired ICE image is compared to a series of "virtual" images based on different registration estimates; those yielding inaccurate virtual images are discarded, and, over time, only accurate estimates remain. EMS is used to inform the algorithm of the change in ICE catheter pose at each time step. EMS measurements are considered differentially to negate the effects of sensor drift, and by doing so we detect changes in the registration parameters without having to repeat or reinitialize the registration process.

\section{Methods}

\subsection{Reference Frames and Transformations}

In Fig. 1(a) we see reference frames assigned to the EMS $(E M)$, ICE catheter tip $(I C)$ and CT/MR-based surface mesh $(C T)$. As described in Sec. 2.2, our algorithm compares ICE data to the surface mesh, thereby implicitly assuming that the mesh represents the left atrium.

We use affine transformations to relate coordinates between reference frames $A$ and $B$ with the following notation:

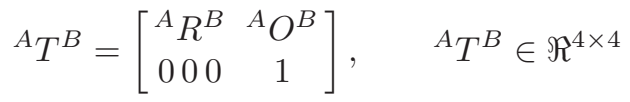

where ${ }^{A} R^{B} \in \Re^{3 \times 3}$ is the coordinate axes rotation offset, and ${ }^{A} O^{B} \in \Re^{3 \times 1}$ is the coordinate origin offset. We represent ${ }^{A} T^{B}$ by its pose vector:

$$
{ }^{A} P^{B}=[x, y, z, \alpha, \beta, \gamma]^{T}, \quad{ }^{A} P^{B} \in \Re^{6 \times 1}
$$

where $[x, y, z]^{T}={ }^{A} O^{B}$ and $(\alpha, \beta, \gamma)$ are the $x y z$ Euler angles describing ${ }^{A} R^{B}$. Finally, we define function $h_{P \rightarrow T}$ to convert ${ }^{A} P^{B}$ into ${ }^{A} T^{B}$ :

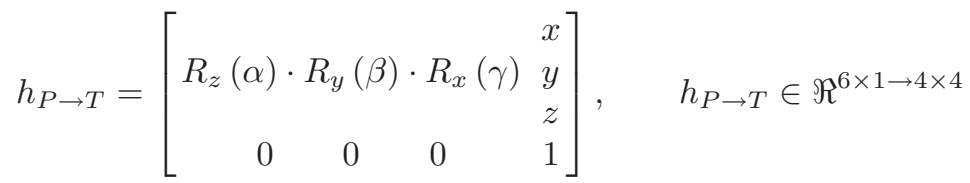

with corresponding inverse $h_{T \rightarrow P}$. 


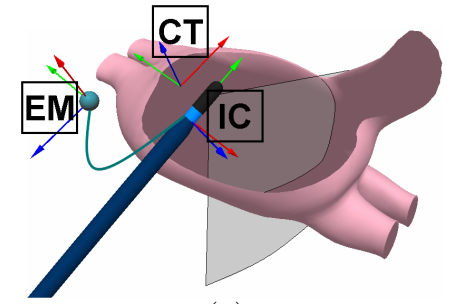

(a)

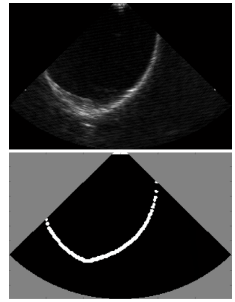

(b)

Fig. 1. In (a) we see a virtual representation of the coordinate axes for the EMS $(E M)$, ICE catheter $(I C)$ and left atrium surface mesh $(C T)$ reference frames. The gray wedge emanating from ICE catheter represents the area imaged under ultrasound. In (b) we see the acquired and virtual ICE images given the catheter state shown in (a).

\subsection{Recursive Bayesian State Estimation}

We employ a Recursive Bayesian State Estimation (RBSE) framework for our incremental registration algorithm. The general RBSE model is as follows: at time step $k$, our estimate of the system's state is defined as $p\left(x_{k} \mid u_{1: k}, z_{1: k}\right)$, the probability distribution over all possible states $x_{k}$ conditioned on all past controls $u_{1: k}$ and measurements $z_{1: k}$ [5]. Here, $x \in \Re^{6 \times 1}$ is the pose of the ICE catheter with respect to the left atrium, $u \in \Re^{4 \times 4}$ is the transformation in ICE catheter pose detected by the EMS, and $z \in \Re^{n \times m}$ is the acquired ICE image of size $n \times m$ pixels. Using reference frame notation, our state and control input are defined as follows:

$$
x_{k}={ }^{C T} P_{k}^{I C} \quad u_{k}={ }^{I C} T_{k-1}^{E M} \cdot{ }^{E M} T_{k}^{I C}
$$

By treating $u_{k}$ as a differential measurement, we can factor out unmodeled drifting of the EMS reference frame provided our sampling frequency is substantially faster than the drifting modes.

At time step $k$, we predict our state evolution using the equation:

$$
\begin{aligned}
x_{k} & =f\left(x_{k-1}, u_{k}, q_{k}\right) \\
& =h_{T \rightarrow P}\left(h_{P \rightarrow T}\left(x_{k-1}\right) \cdot{ }^{I C} T_{k-1}^{E M} \cdot{ }^{E M} T_{k}^{I C}\right)+q_{k}
\end{aligned}
$$

with process noise $q_{k} \in \Re^{6 \times 1}$, and estimate our measurement as:

$$
y_{k}=g_{i c e}\left(x_{k}, r_{k}\right), \quad y_{k} \in \Re^{n \times m}
$$

with measurement noise $r_{k} \in \Re^{n \times m}$. Function $g_{i c e}$ generates a virtual ICE image $\left(y_{k}\right)$ of the surface mesh as it would appear if taken from pose $x_{k}$ (Fig. 1(b)]. Acquired ICE image $z_{k}$ is compared to $y_{k}$ using a normalized mutual information (NMI) correlation metric defined in [6].

Because Eqns. 5 and [6] are non-linear, and because Eqn. 6] cannot be defined analytically, we use the UPF as our RBSE technique. The UPF is useful in state estimation problems that are highly nonlinear and do not yield Gaussian 


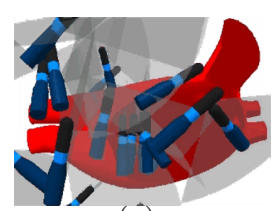

(a)

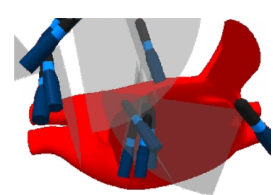

(b)

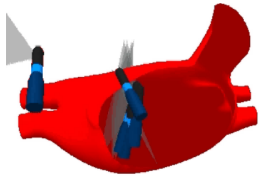

(c)

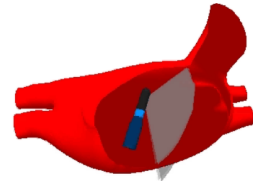

(d)

Fig. 2. We initially consider a uniform sampling of all possible estimates (a), but as we consider more ultrasound data in (b) and (c) we discard inaccurate estimates until we have converged upon a solution (d)

state posteriors [7]. Instead, the UPF represents state posteriors using several "particles" sampled over the distribution.

Each particle in our UPF implementation is initially assigned a random pose $x_{0}={ }^{C T} P^{I C}$ taken from a uniform distribution over all possible poses, the range of which is assumed to be slightly larger than the size of an adult left atrium:

$$
\begin{aligned}
\text { Range }\left({ }^{C T} P^{I C}\right) & =\text { Range }\left([x, y, z, \alpha, \beta, \gamma]^{T}\right) \\
& =\left[ \pm 50 \mathrm{~mm}, \pm 50 \mathrm{~mm}, \pm 50 \mathrm{~mm}, \pm 180^{\circ}, \pm 90^{\circ}, \pm 180^{\circ}\right]^{T}
\end{aligned}
$$

After all particles have undergone state evolution, they are weighted using their NMI correlation score and resampled using stochastic universal sampling [8]. Particles yielding virtual ICE images with low correlation scores are discarded and eventually only a few particles, whose estimates are close to the actual state, remain in consideration (Fig. 21). To speed up the process, we convert ICE images to binary, so that all comparisons between real and virtual images can be performed using bit-wise operations. We also decimate the resolution of the acquired ICE image when there are a large number of particles to consider, and gradually return to the native resolution as we converge upon a solution.

\subsection{EMS to Mesh Registration}

Our UPF implementation generates an estimate of ${ }^{C T} P^{I C}$, but our ultimate goal is to recover ${ }^{C T} T_{k}^{E M}$. We can do this in one of two ways. In general, we assume that ${ }^{C T} T^{E M}$ may change over time; that is, the EMS reference frame may move without our knowledge. In this case we use the equation:

$$
{ }^{C T} T_{k}^{E M}=h_{P \rightarrow T}\left({ }^{C T} P_{k}^{I C}\right) \cdot{ }^{I C} T_{k}^{E M}
$$

However, if we know that ${ }^{C T} T^{E M}$ will only change very slowly, we can compute a least-squares estimate of ${ }^{C T} T_{k}^{E M}$ by considering the last $n$ UPF estimates simultaneously. Increasing $n$ leads to a more stable estimate, but at the expense of increased sensitivity to EMS reference frame drifting.

\subsection{Experimental Setup}

We verified our registration algorithm's performance using a left atrium phantom cast in a silicone compound that readily images under ultrasound. We rotated 


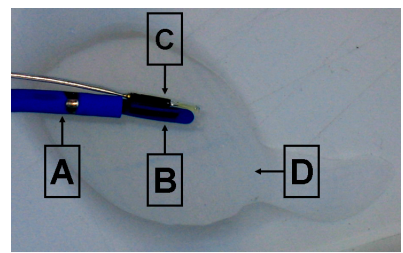

(a)

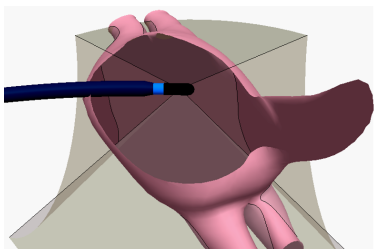

(b)

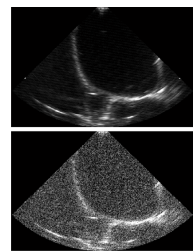

(c)

Fig. 3. In (a) we see the guide catheter A) with ICE catheter tip B) emerging from its lumen. A 6DOF Ascension sensor C) is mounted to the ICE catheter, which images the left atrium D) phantom below. In (b) we see the imaged volume swept out by rotating the ICE catheter while continuously acquiring data. In (c) we see an acquired "clean" ICE image on top, and its "noisy" counterpart on bottom.

an ICE catheter about its longitudinal axis while continuously acquiring crosssectional images of the phantom. The catheter was rotated using a motorized system that commanded a $60^{\circ}, \frac{1}{12} H z$ sine wave trajectory. Images were acquired at $10 \mathrm{~Hz}$, or roughly every $2^{\circ}$ at the peak rotation velocity. We purposefully chose not to calibrate the ICE catheter transducer, instead opting to treat spatial distortions in its images as a source of measurement noise.

We mounted a 6DOF position/orientation sensor (Ascension Technology Corp., Burlington, VT) to the ICE catheter's tip to monitor its exact position and orientation over time. A second sensor was affixed to the phantom at a known position and orientation allowing us to measure ${ }^{C T} T^{E M}$ for validation. Fig. 3(a) shows the components used in our experimental setup. We see a close-up of the ICE catheter tip in position for imaging the left atrium phantom. Fig. 3(b) shows the imaged volume swept out by rotating the ICE catheter while acquiring data.

In addition to testing the algorithm's accuracy under the stationary conditions described above, we performed a separate test where we slowly moved the EMS reference frame by translations of $\leq 20 \mathrm{~mm}$ along each axis, and rotations of $\leq 10^{\circ}$ about each axis, to emulate sensor drift caused by breathing or patient movement. The translation drift speed was capped at $\frac{1 \mathrm{~mm}}{\mathrm{sec}}$, and rotation

Table 1. Registration Errors with stationary EMS reference frame

\begin{tabular}{l||l|l|l|l|l}
\hline Measured Error & S Sweep & 2 Sweeps & 3 Sweeps & 4 Sweeps \\
\hline \hline "Clean" Position Error & $\mu=3.38 \mathrm{~mm}$ & $\mu=1.01 \mathrm{~mm}$ & $\mu=0.80 \mathrm{~mm}$ & $\mu=0.74 \mathrm{~mm}$ \\
& $\sigma=2.33 \mathrm{~mm}$ & $\sigma=0.56 \mathrm{~mm}$ & $\sigma=0.39 \mathrm{~mm}$ & $\sigma=0.33 \mathrm{~mm}$ \\
"Clean" Orientation Error & $\mu=12.3^{\circ}$ & $\mu=2.77^{\circ}$ & $\mu=2.33^{\circ}$ & $\mu=2.22^{\circ}$ \\
& $\sigma=8.21^{\circ}$ & $\sigma=1.63^{\circ}$ & $\sigma=0.90^{\circ}$ & $\sigma=0.84^{\circ}$ \\
\hline \hline
\end{tabular}


drift speed at $\frac{1^{\circ}}{s e c}$. Finally, to ensure the robustness of our NMI-based image correlation algorithm, we performed a third test where we added Gaussian noise ( $\mu=0.2, \sigma=0.25)$ to the acquired ICE images before thresholding to binary (Fig. 3(c) . The amount of noise added was chosen based on the threshold after which ultrasound reflections were completely obscured in the images.

\section{Results}

\subsection{Stationary EMS Reference Frame}

Holding the EMS reference frame stationary, we tested our registration algorithm's accuracy as the ICE catheter was rotated through its full extent four times (over $48 \mathrm{sec}$ ). We computed a least squares estimate of ${ }^{C T} T_{k}^{E M}$ and measured the accuracy of our estimate after each sweep over 100 separate trials. We performed this experiment twice, once using "clean" ICE images and once using "noisy" ICE images. In Tbl. 1] we show the mean and standard deviation of error in the registration algorithm's estimate after each sweep. We compute two quantities: registration position error and orientation error. The position error is measured as the Euclidean distance between the actual and estimated coordinate origin offset, $\left\|{ }^{C T} O_{\text {act }}^{E M}-{ }^{C T} O_{\text {est }}^{E M}\right\|$, and the orientation error is measured as the minimum angle required to transform ${ }^{C T} R_{\text {est }}^{E M}$ into ${ }^{C T} R_{\text {act }}^{E M}$ using an angle-axis rotation scheme. We see that our accuracy improves with continued imaging, and, using both "clean" and "noisy" images, our estimate's error falls below $2 \mathrm{~mm}$ and $6^{\circ}$ after considering four sweeps of data.

\subsection{Drifting EMS Reference Frame}

Next we tested the registration algorithm's accuracy while moving the EMS reference frame as described in Sec. 2.4. We computed ${ }^{C T} T_{k}^{E M}$ using Eqn. 8 and measured the accuracy of our estimate over 100 separate trials. This test was performed once using "clean" ICE images and once using "noisy" ICE images.

In Fig. 4 we see two plots charting the average position and orientation errors of our registration estimate over time. The duration shown is equivalent to two rotation sweeps. We see that after one sweep, the mean position error drops below $5 \mathrm{~mm}$ in both the "clean" and "noisy" case, and after two sweeps the position error stays roughly around $2.5 \mathrm{~mm}$. Similarly, the orientation error drops below $10^{\circ}$ after one sweep, and below $5^{\circ}$ after two sweeps. We compared our results to a "static" estimation scheme, where ${ }^{C T} T_{k}^{E M}$ is assumed constant over time. We set the static scheme's estimate to ${ }^{C T} T_{a c t_{0}}^{E M}$, so that initially there is no error in its estimate. As the EMS reference frame drifts back and forth, the static scheme's performance is inversely proportional to the amount of drift. We see that by the end of two sweeps, the average position error in the static estimate is greater than $15 \mathrm{~mm}$. This would be worse if the static scheme's estimate was not initialized to ${ }^{C T} T_{\text {act }_{0}}^{E M}$. 


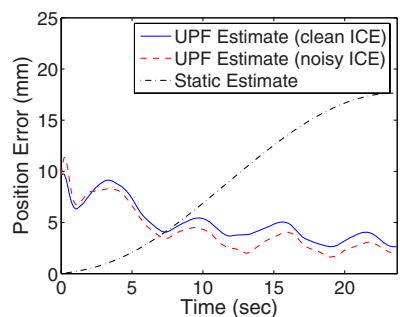

(a)

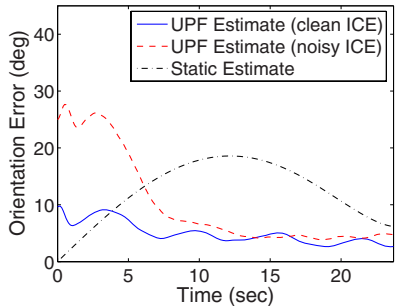

(b)

Fig. 4. Registration Errors with moving EMS reference frame

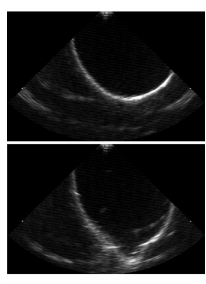

(a)

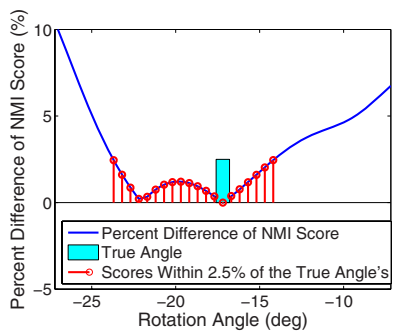

(b)

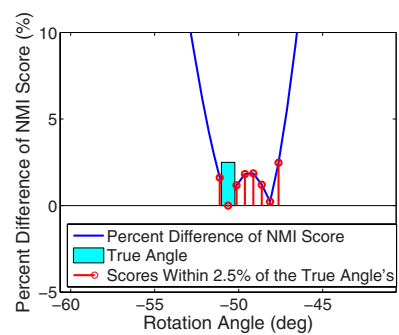

(c)

Fig. 5. In (a) we see ICE images of the anatomical center (top) and the pulmonary veins (bottom). We compute the NMI score percent-differentials for neighboring angles that also image (b) the anatomical center and (c) the veins.

\subsection{Performance Limitations}

Our ability to estimate the correct registration parameters is not only limited by the resolution of our NMI correlator, but also by the region of the anatomy we are currently imaging. In Fig. 5 we highlight our uncertainty when imaging two different regions of the anatomy. When imaging the mostly-uniform anatomical center, the range of angles generating NMI scores within $2.5 \%$ of the score at the true orientation angle is $10^{\circ}$. When imaging the feature-rich region surrounding the veins, however, this range drops to $5^{\circ}$. Consequently, our uncertainty increases when the ICE catheter sweeps through the anatomical center, qualifying the oscillatory behavior we see in our estimate error (Fig. 4). Figs. 5(b) and $5(\mathrm{c})$ also reveal that our NMI correlator can suffer from local minima. We avoid moving towards these minima based on knowledge of our current estimate and rotation command. However, we may get trapped by a minimum if it is closer to our current estimate than the true state.

\section{Discussion}

We have presented a novel method for incrementally registering EMS data to CT using 2D ICE image slices, rather than a 3D ICE volume reconstruction. The advantage of the incremental approach is that we are robust to sensor drift and small patient movements. Using a left atrium phantom, our algorithm estimates 
registration parameters with high precision after considering less than a minute's worth of data. Our "virtual" ICE image generation and NMI correlation scheme is efficient and robust to unbiased noise.

A limitation to our approach is that our confidence varies in accordance to the "feature-richness" of the anatomy we are currently imaging. A solution might be to consider the last $n$ ICE images at each time step, increasing the overall featurecontent under consideration. The downside of this strategy would be increased sensitivity to sensor drift or patient motion. However, assuming that unmodeled drifts are "slow-moving", we can counteract this sensitivity by considering only a few samples of the most recently-acquired data.

\section{References}

1. Sun, Y., Kadoury, S., Li, Y., John, M., Resnick, J., Plambeck, G., Liao, R., Sauer, F., Xu, C.: Image guidance of intracardiac ultrasound with fusion of pre-operative images. In: Medical Image Computing and Computer-Assisted Intervention, pp. 60$67(2007)$

2. Wein, W., Khamene, A., Clevert, D.A., Kutter, O., Navab, N.: Simulation and fully automatic multimodal registration of medical ultrasound. In: Medical Image Computing and Computer-Assisted Intervention, pp. 136-143 (2007)

3. Zhong, H., Kanade, T., Schwartzman, D.: Sensor guided ablation procedure of left atrial endocardium. In: Medical Image Computing and Computer-Assisted Intervention, pp. 1-8 (2005)

4. Zhong, H., Kanade, T., Schwartzman, D.: Virtual touch: An efficient registration method for catheter navigation in left atrium. In: Medical Image Computing and Computer-Assisted Intervention, pp. 437-444 (2006)

5. Thrun, S.: Probabilistic algorithms in robotics. AI Magazine 21(4), 93-109 (2000)

6. Pluim, J.P., Maintz, J.A., Viergever, M.A.: Mutual-information-based registration of medical images: A survey. IEEE Transactions on Medical Imaging 22(8), 986-1004 (2003)

7. Rui, Y., Chen, Y.: Better proposal distributions: object tracking using unscented particle filter. In: Proceedings of the IEEE Computer Society Conference on Computer Vision and Pattern Recognition, vol. 2, pp. 786-793 (2001)

8. Baker, J.E.: Reducing bias and inefficiency in the selection algorithm. In: Proceedings of the Second International Conference on Genetic Algorithms and their Application, pp. 14-21 (1987) 